\title{
Implications of high power losses in IR femtosecond laser inscribed fibre Bragg gratings
}

\author{
K.T. O'Mahoney ${ }^{a}$, A.S. Main ${ }^{b}$, D.J. $\mathrm{Webb}^{b}$, A. Martinez ${ }^{b}$ and D.A. Flavin ${ }^{a}$ \\ ${ }^{a}$ Optics Research Group, Department of Computing, Mathematics and Physics, Waterford \\ Institute of Technology, Waterford, Ireland \\ ${ }^{b}$ Photonics Research Group, School of Engineering and Applied Science, Aston University, \\ Aston, Birmingham, U.K., B4 7ET
}

\begin{abstract}
We report on high power issues related to the reliability of fibre Bragg gratings inscribed with an infrared femtosecond laser using the point-by-point writing method. Conventionally, fibre Bragg gratings have usually been written in fibres using ultraviolet light, either holographically or using a phase mask. Since the coating is highly absorbing in the UV, this process normally requires that the protective polymer coating is stripped prior to inscription, with the fibre then being recoated. This results in a time consuming fabrication process that, unless great care is taken, can lead to fibre strength degradation, due to the presence of surface damage. The recent development of FBG inscription using NIR femtosecond lasers has eliminated the requirement for the stripping of the coating. At the same time the ability to write gratings point-by-point offers the potential for great flexibility in the grating design. There is, however, a requirement for reliability testing of these gratings, particularly for use in telecommunications systems where high powers are increasingly being used in long-haul transmission systems making use of Raman amplification. We report on a study of such gratings which has revealed the presence of broad spectrum power losses. When high powers are used, even at wavelengths far removed from the Bragg condition, these losses produce an increase in the fibre temperature due to absorption in the coating. We have monitored this temperature rise using the wavelength shift in the grating itself. At power levels of a few watts, various temperature increases were experienced ranging from a few degrees up to the point where the buffer completely melts off the fibre at the grating site. Further investigations are currently under way to study the optical loss mechanisms in order to optimise the inscription mechanism and minimise such losses.
\end{abstract}

\section{INTRODUCTION}

In fibre Bragg gratings (FBGs) have been one of the most exciting developments in the telecommunications and optical sensing fields in recent years. In the optical sensing field they have proved to be one of the most promising candidates for use as embedded sensors in fibre-optic smart structures. The measured quantity is the peak reflective wavelength of the grating. In the telecommunications field FBGs have been used for applications such as wavelength division multiplexing, narrow band tunable filters and for dispersion compensation. Ideally for such applications the temperature sensitivity of the gratings needs to be mitigated and can be nearly eliminated by embedding the grating in another substance. Power levels in telecom systems have increased as the demand for greater bandwidth increases. Therefore the resonant wavelength's stability when used in high power applications assumes a greater significance.

The principle mechanism involved in the inscription process of standard fibre Bragg gratings is the photosensitivity of Ge-doped fibre to UV light. ${ }^{1}$ Reliability issues are a concern for UV inscribed FBGs as in the writing process there is the requirement that the buffer is stripped from the fibre, because of a sensitivity to UV light. This can result in surface strength degradation, the extent of which depends on the stripping mechanism used. Hydrogen loading to increase the photosensitivity of the fibres has also been shown to decrease the mechanical strength of the fibre. ${ }^{2}$

The development of near infrared point by point inscription techniques for fibre Bragg gratings by femtosecond laser pulses has eliminated the requirement for, not only stripping off the buffer coating, but also the need for a phase mask and photosensitised fibre. ${ }^{3}$ There is therefore the potential for this writing technique to significantly

Reliability of Optical Fiber Components, Devices, Systems, and Networks III, edited by Hans G. Limberger, M. John Matthewson,

Proc. of SPIE Vol. 6193, 61930Z, (2006) - 0277-786X/06/\$15 - doi: 10.1117/12.662901

Proc. of SPIE Vol. 6193 61930Z-1 
reduce fibre Bragg grating production time and associated costs. Femtosecond laser inscribed gratings also exhibit characteristics such as high thermal stability, large refractive index modulation and confinement of the grating within a fraction of the fibre core. ${ }^{4}$ However, as with inscription of gratings by UV light there are also reliability issues with inscription by femtosecond lasers which need to be addressed. The refractive index changes induced when inscribing FBGs with an IR femtosecond laser are thought to be due to a densification of the irradiated region. ${ }^{5}$ The endurance of fibre, which has been subjected to this type of damage, to operate normally under conditions where it is being subjected to high powers, needs to be evaluated.

Damage to fibres when used in high power applications has been observed. For example the fibre fuse phenomenon ${ }^{6}$ has been observed with measurement of the threshold for fibre fuse to occur, the effects of end-face damage under high powers, ${ }^{7}$ and for damage to occur to the fibre coating. ${ }^{8}$ The fibre coating damage can be caused by light leaking from the fibre, when it is broken or bent in a high power environment, such as occurs with the introduction of Raman amplifier pump lasers. This light generates heat and may possibly ignite the buffer. $^{9}$

In this work we present observations on temperature increases in infrared inscribed FBGs when subjected to high powers. We apply a drift compensated high resolution interferometric wavelength shift detection technique for grating demodulation ${ }^{10}$ and record the wavelength shift of a grating under high power on an Optical Spectrum Analyser for comparison.

\section{MEASUREMENT CONSIDERATIONS}

\subsection{Fibre Bragg Gratings}

Light which is launched down a fibre and which matches the resonant wavelength of the grating, $\lambda_{B}$, experiences strong reflection for the condition that, ${ }^{11}$

$$
\lambda_{B}=\frac{2 n \Lambda}{N}
$$

where $\mathrm{n}=$ the effective mode index, $\Lambda=$ spatial periodicity of the grating and $\mathrm{N}$ the order of diffraction. The measured quantity, the peak reflected wavelength or dip in transmission, changes as the grating is subjected to temperature, pressure or mechanical strain, ${ }^{12}$

$$
\frac{d \lambda_{B}}{d X}=2\left(\Lambda \frac{d n}{d X}+n \frac{d \Lambda}{d X}\right): X=T, P, \delta l
$$

Typical temperature to wavelength shifts for FBG's at $1550 \mathrm{~nm}$ are $\sim 10-13 \mathrm{pm} /{ }^{\circ} \mathrm{C}$

\subsection{Mach Zehnder Interferometer}

The component of the interferogram generated by an un-balanced Mach Zehnder interferometer which is reflected by a fibre Bragg grating can be represented as a sinusoidally varying signal; a strain induced change in the reflected wavelength, $\delta \lambda$, manifests itself as a change in phase, $d \psi$, of this reflected signal, given by ${ }^{10}$

$$
d \psi=-\frac{2 \pi n d}{\lambda^{2}} d \lambda
$$

where nd is the optical path difference in the interferometer. From equation (3) a temperature induced phase change becomes:

$$
\delta \psi=-\frac{2 \pi n d}{\lambda} \xi \delta T
$$

where $\delta T$ is the change in temperature of the grating and $\xi$ is the normalised temperature to wavelength shift responsivity of the grating, ${ }^{13}$

$$
\xi=\frac{1}{\lambda_{s}} \frac{\delta \lambda}{\delta T}
$$

which has a value of $6.67 \times 10^{-6} /{ }^{\circ} \mathrm{C}$ 


\section{EXPERIMENTAL CONFIGURATION}

Two experimental configurations were used to record the effects of high power Raman amplifier pump lasers on femtosecond inscribed FBG's.

\subsection{Mach Zehnder Interferometer}

In the experimental configuration shown in fig. 1, an unbalanced Mach-Zehnder interferometer (MZI) is illuminated by a $1550 \mathrm{~nm}$ broadband source. One arm of the interferometer contains a $\mathrm{LiNbO}_{3}$ phase modulator. The second arm contains an air gap with which the non-zero optical path difference, OPD, between the two arms of the interferometer can be adjusted. By adjusting the path difference in the arms of the interferometer we adjust the free spectral range of the MZI. The free spectral range of the interferometer was set to $\sim 2 \mathrm{~nm}$, giving an OPD in the interferometer of $\sim 1.2 \mathrm{~mm}$. The interferometer is repetitively scanned over it's free spectral range by applying a serrodyne waveform to the phase modulator.

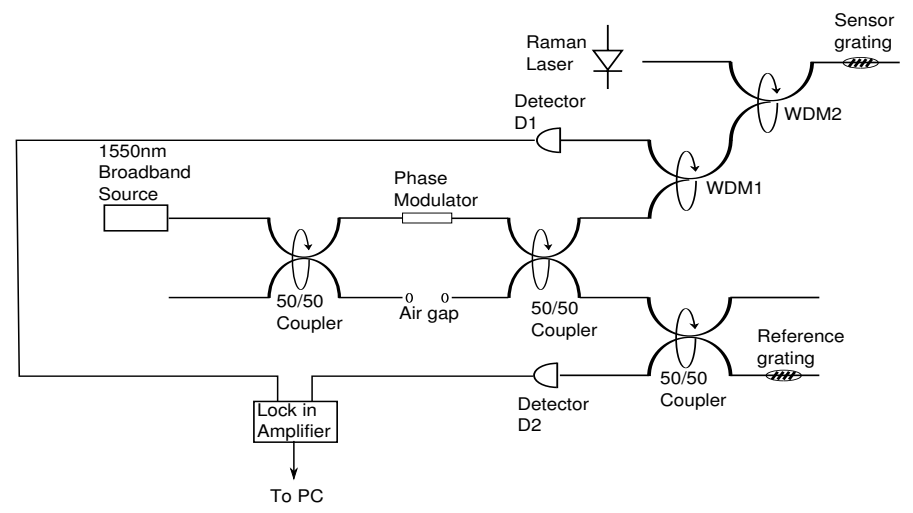

The sensing grating is connected to one of the output arms of the interferometer via 2 add/drop multiplexers, the first of which allows detection of the reflected signal from the grating at detector D1 and the second allows injection of light from the high power Raman laser. A reference grating is connected to the second output arm of the interferometer via a 50/50 coupler to allow detection of the reflected signal at detector D2. If the optical path imbalance between the arms of the interferometer is shorter than the coherence length of the gratings a sinusoidal signal will be reflected from the gratings. The wavelength of the sensing grating is $\sim 1549 \mathrm{~nm}$. Therefore, from equation (4), the temperature to phase shift response in this case is $\sim 1.6 \mathrm{deg} /{ }^{\circ} \mathrm{C}$. The signals captured at photodiodes D1 and D2 are monitored using a lock-in amplifier to detect any phase change between the reference and sensor gratings which is acquired by a PC.

A series of femtosecond inscribed Bragg gratings are tested for damage when high powers are being transmitted down the fibre by replacing the sensing grating with a series of femtosecond inscribed gratings of varying lengths and reflectivities.

\subsection{Optical Spectrum Analyser}

In the second configuration used to demodulate the gratings, illustrated in figure(2), light from a 1550nm SLED is launched through $2 \times 50 / 50$ couplers. The Raman laser is launched through the input arm of the second coupler. A femtosecond inscribed Bragg grating is spliced to the output arm of this coupler and the component of light from the $1550 \mathrm{~nm}$ SLED which is reflected by the grating propagates back through the two couplers and is detected by the OSA. The Raman laser power is increased from $0-4.5 \mathrm{~W}$ and the

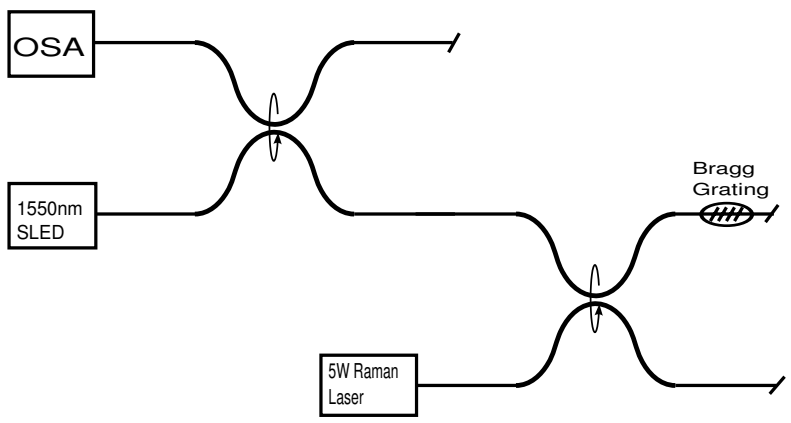
wavelength shift due to temperature increases experienced by the grating is recorded by the OSA. 


\section{RESULTS AND DISCUSSION}

\subsection{Interferometric Analysis}

\subsubsection{Transmitted Power Losses}

Figure 3 shows the transmitted power losses through 5 IR inscribed gratings. The insertion losses for the 5 femtosecond laser inscribed gratings, 3 written in standard fibre, labeled ST, and 2 written in BT telecom fibre, at the Raman Laser output of $1455 \mathrm{~nm}$ vary significantly, from $\sim 1.5-\sim 3 d B$.

\subsubsection{Temperature Increases}

In figure (4), the phase change in degrees is plotted for 4 gratings when subjected to laser powers of $0-4.5 \mathrm{~W}$. From equation (3), the approximate change in temperature recorded by the individual gratings is plotted on the right hand side and range from $43^{\circ} \mathrm{C}$ to $162^{\circ} \mathrm{C}$ (-70 to -260 deg phase change) at $4.5 \mathrm{~W}$. A temperature calibration of the gratings gave a wavelength shift of $\sim 11.5 \mathrm{pm} /{ }^{\circ} \mathrm{C}$. The specifications for the gratings illustrated in figure (4) were

$\begin{array}{llc}\text { Grating } & \text { Length } & \text { Reflectivity } \\ \text { ST6 } & >10 \mathrm{~mm} & 3.5 \mathrm{~dB} \\ \text { ST9 } & 10 \mathrm{~mm} & 8 \mathrm{~dB} \\ \text { ST10 } & 5 \mathrm{~mm} & 8 \mathrm{~dB} \\ \text { ST12 } & 5.5 \mathrm{~mm} & 5 \mathrm{~dB}\end{array}$

From examination of figure (3), grating ST10, which exhibited a similar insertion loss to grating ST9, is shown in figure (4) to exhibit the lowest temperature increase.

Grating ST6, the longest grating, but with one of the lowest reflectivities, exhibited the largest observed phase change of $\sim 260^{\circ} \mathrm{C}$ at $4.5 \mathrm{~W}$ without the buffer melting off the fibre. This phase change corresponds to a temperature increase of $\sim 162{ }^{\circ} \mathrm{C}$ at a temperature to phase shift response of $\sim 1.6 \mathrm{deg} /{ }^{\circ} \mathrm{C}$.

Grating ST9, the second longest of the gratings but with one of the highest reflectivities had the buffer melted off the fibre at $\sim 89^{\circ} \mathrm{C}$. The apparently low temperature recorded for the melting point of the buffer, in comparison to the temperature reached by grating ST6, suggested that the experimental configuration above was not recording the actual change in temperature experienced by the grating.

\subsubsection{Damage}

To assess whether there was any damage being done to the grating at high powers the power levels to which the fibre was exposed in the earlier tests were individually switched on and then off. The wavelength at which the grating started from and returned to was then recorded by the PC. An examination of figure (5) shows that grating BT2 returned to its original wavelength after exposure to power levels from $0-4.5 \mathrm{~W}$. Similar tests on the gratings whose buffers did not melt off showed the same result, they all returned to their original wavelengths. However, examination of the full scan taken of grating $S T 9$ (figure (6)), whose buffer appeared to strip off at 


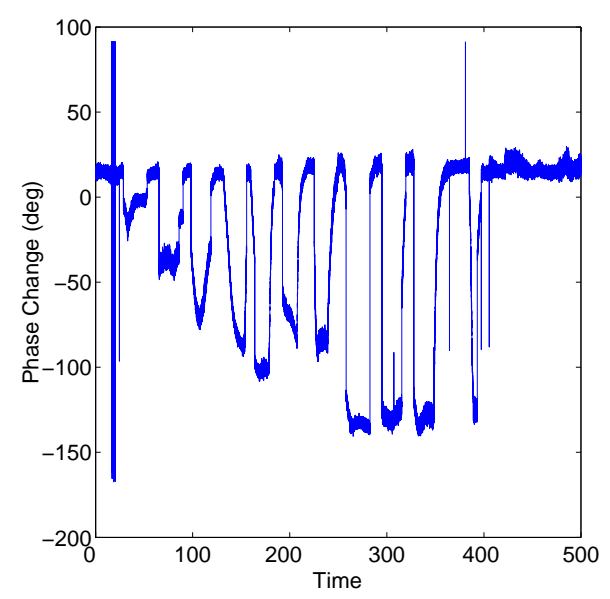

Figure 5. Phase change of grating BT2 with increase in laser power from 0 to $4.5 \mathrm{~W}$

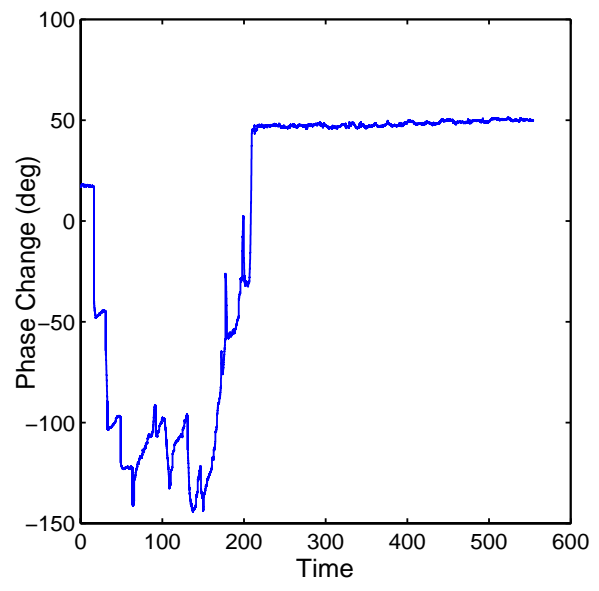

Figure 6. Phase change of grating ST9 with increase in laser power from 0 to $4.5 \mathrm{~W}$

$\sim 89^{\circ} \mathrm{C}$, where the power had been increased to $4.5 \mathrm{~W}$ after the buffer had stripped off shows that there was a phase change between the recorded starting and finishing phase difference.The $29^{\circ}$ change in phase equates to a $0.1 \mathrm{~nm}$ change in the original wavelength.

\subsection{OSA Observations}

\subsubsection{Spectral Broadening}

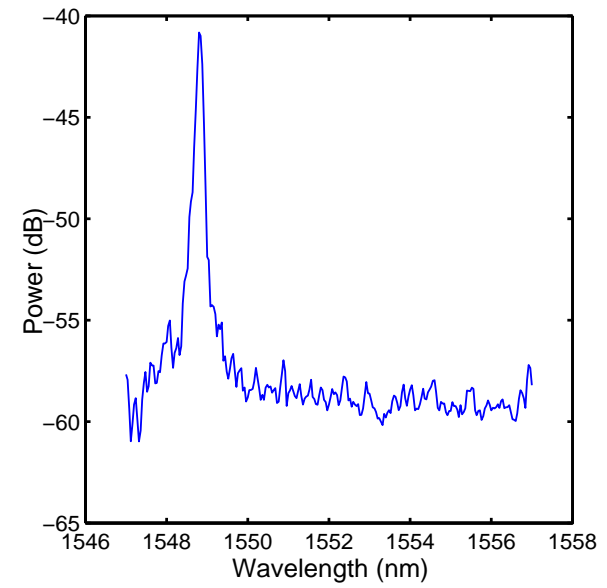

Figure 7. Grating with no Raman power launched down fibre.

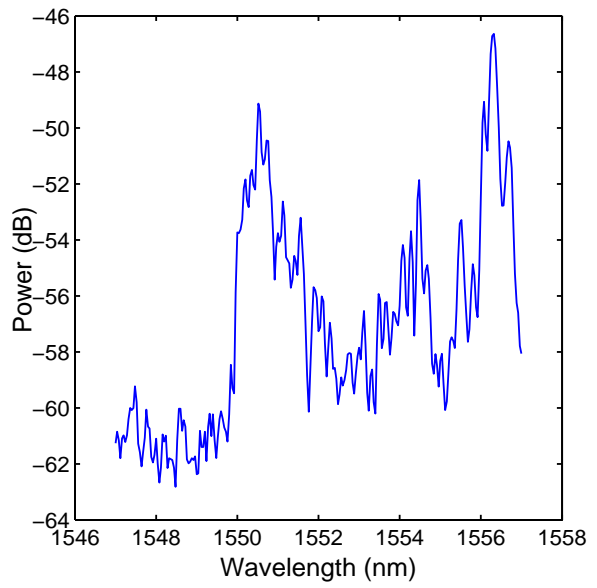

Figure 8. Spectrum recorded by OSA at buffer melting point of $2.5 \mathrm{~W}$

Examination of a grating with an OSA, clearly shows the spectrum of the grating being broadened as the laser power level through the fibre is increased. Figure (7) illustrates the spectrum for a grating with the Raman laser switched off. Figure (8), shows the spectrum of this grating with $2.5 \mathrm{~W}$ launched through the fibre. At this power level the buffer had melted off the fibre around this grating.

The wavelength shift of the spectral content of the grating due to the temperature increase at melting point is more significant than that recorded using the interferometric configuration. The spectral broadening observed 
for this grating covers $\sim 7 \mathrm{~nm}$. ; this is far beyond the FSR of the interrogating interferometer and hence the readings produced by that system are not reliable. A wavelength shift of $\sim 7 \mathrm{~nm}$, at a sensitivity of $\sim 11 \mathrm{pm} /{ }^{\circ} \mathrm{C}$ would correspond to an apparent temperature increase of $\sim 540^{\circ} \mathrm{C}$-certainly more than enough to melt the buffer coating.

\subsubsection{Damage}

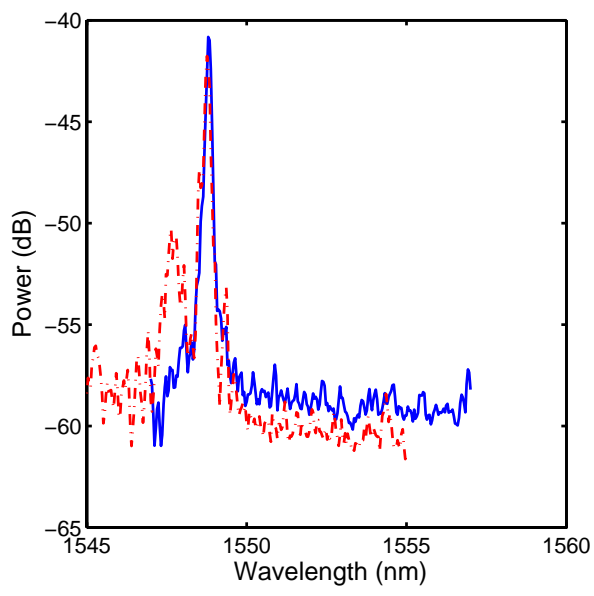

Figure 9. Comparison of spectra (no power) before (continuous line) and after (dash dot) melting of buffer

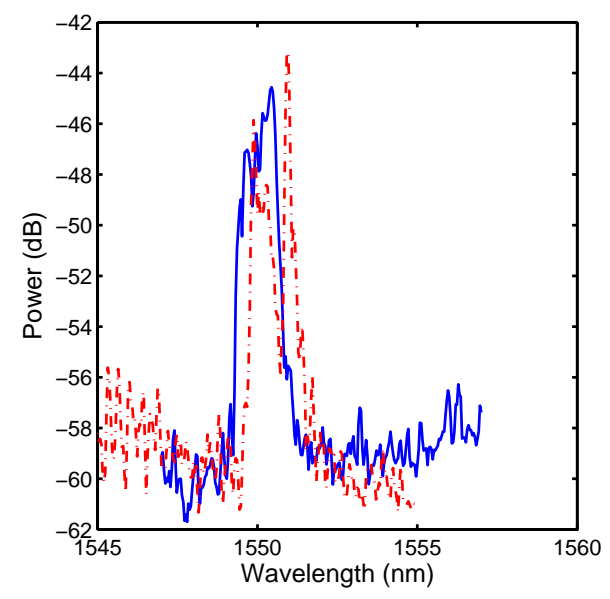

Figure 10. Comparison of spectra of the grating before (continuous line) and after (dash-dot) at $1.135 \mathrm{~W}$

An examination of the spectrum of the grating after melting of the buffer with the Raman laser switched off, figure (9), shows that there is extra spectral content on both sides of the grating spectrum. The addition of this spectral content, or chirping of the grating was also reflected in the phase measurements recorded by the MZI.

The wavelength shift experienced by the grating doesn't diminish with the removal of the buffer. Figure (10) shows the spectra of the grating before (continuous line) and after (dash-dot) melting of the buffer when 1.135 $\mathrm{W}$ of Raman power is launched through the fibre.

\section{CONCLUSION}

We have investigated the effects of broad spectrum losses across NIR femtosecond laser inscribed gratings. When high powers are used, even at wavelengths far removed from the Bragg condition, these losses produce an increase in the fibre temperature due to absorption in the coating. We have monitored this temperature rise using the wavelength shift in the grating itself. At powers of a few watts, various temperature increases were experienced ranging from a few degrees up to the point where the buffer completely melts off the fibre at the grating site. The high temperatures can induce a permanent change in the grating profile. Further investigations are currently under way to fully understand the loss mechanism and to to optimise the inscription mechanism and minimise such losses, which are not necessarily intrinsic to the femtosecond writing mechanism. The work demonstrates the need to have tight control over the grating quality in high power applications. 


\section{REFERENCES}

1. Y.J.Rao, "Recent progress in applications of in-fibre bragg grating sensors," Optics and Lasers in Engineering 31, pp. 297-324, 1999.

2. C. Wei, C. Ye, S. James, R. Tatam, and P. Irving, "The influence of hydrogen loading and the fabrication process on the mechanical strength of optical fibre bragg gratings," Optical Materials 20, pp. 241-251, 2002.

3. A.Martinez, M.Dubov, I.Khrushchev, and I.Bennion, "Direct writing of fibre bragg gratings by femtosecond laser," Electronic Letters 40, September 2004.

4. A.Martinez, Y.Lai, M.Dubov, I.Y.Khrushchev, and I.Bennion, "Vector bending sensors based on fibre bragg gratings inscribed by infrared femtosecond laser," Electronics Letters 41(8), 2005.

5. D.Homoelle, S.Wielandy, and A. L.Gaeta, "Infrared photosensitivity in silica glasses exposed to femtosecond laser pulses," Optics Letters 24, pp. 1311-1313, September 1999.

6. D.P.Hand and P.S.Russell, "Solitary thermal shock waves and optical damage in optical fibres - the fibre fuse," Optics Letters 13, pp. 767-769, Sep 1988.

7. K. Seo, N. Nishimura, and M. Shiino, "Evaluation of high-power endurance in optical fiber links," Furukawa Review (24), 2003.

8. R. Percival, E. Sikora, and R. Wyatt, "Catastrophic damage and accelerated ageing in bent fibres caused by high optical powers," Electronics Letters 36, March 2000.

9. E. Sikora, D. McCartney, K. Farrow, and R. Davey, "Reduction in fibre reliability due to highoptical power," Electronics Letters 39, July 2003.

10. A. D. Kersey and T. A. Berkoff, "Fiber-optic bragg grating strain sensor with drift-compensated highresolution interferometric wavelength-shift detection," Optics Letters 18, January 1993.

11. K. O. Hill and G. Meltz, "Fiber bragg grating technology fundamentals and overview," Journal of Llightwave Technology, 15, August 1997.

12. Y.-J. Rao, "In fibre bragg grating sensors," Meas. Sci. Technol. 8, pp. 355-375, 1997.

13. S. Abbenseth and S. Lochmann, "Distinct enlargement of network size or measurement speed for serial fbg sensor networks utilizing sik-ds-cdma," Journal of Physics Conference Series 15, pp. 149-154, 2005. 